\title{
AN UML-BASED META-LANGUAGE FOR THE QOS-AWARE ENTERPRISE SPECIFICATION OF OPEN DISTRIBUTED SYSTEMS
}

\author{
B. El Ouahidi, M. Bouhdadi, D.Bourget \\ ENST-Bretagne, Département informatique Technopôle Brest Iroise, BP. 832 Brest FRANCE \\ \{Bouabid.Ouahidi, Mohamed.Bouhdadi,Daniel.Bourget\}@enst-bretagne.fr
}

\begin{abstract}
We address in this paper the need to develop appropriate notations for the QoSaware specification of the ODP (Open Distributed Processing) enterprise viewpoint. The Unified Modelling Language (UML) is currently emerging as de facto standard for formal object-oriented modelling. Several approaches are being used in the field of the formalization of UML. For several reasons, we use the metamodelling approach in our work. We analyze the QoS concepts defined in QoS foundation modelling concepts part and in the QoS in enterprise specification part. The result is an UML meta-model of the core QoS concepts and their relationships, which is considered as the abstract syntax of a concrete language for QoS-aware ODP enterprise specifications.
\end{abstract}

\section{INTRODUCTION}

ODP (ISO, 1994) systems create important requirements for communication systems and end systems since they require much higher rates and much sever temporal constraints than classical systems ; in particular ODP systems with multimedia components (stream interactions, flow interfaces). Over the past several years there has been a considerable amount of research within the field of quality of service support for distributed multimedia systems. Most of the work has been occurred within the context of individual architectural layers such as the distributed system platform (Vogel, 1994), (Mullender, 93), (APM, 91), (Coulson, 92), (Nicolaou, 90), operating system (Stankovics, 95), (Coulson, 95), (Feldmeir, 93), (Bulterman, 91), transport subsystem (Campbell, 92), (Shulzrine 1997), (Barden 1997 RSVP), (Doeringer, 90) and network (DiffServ, 98), (IntServ, 94). However, in order to meet the service user QoS requirements, all system components involved in the QoS provision should support a level of QoS in a way that the required end-toend QoS is satisfied. In recognition of this, a number of QoS architectures have proposed to provide QoS guarantees. Elsewhere, in order for to QoS guarantees to be effective, QoS aspects have to be considered in the overall process of development. This have led to the definition of the framework for QoS in ODP (ISO, 98) whose the objectives are to extend the existing QoS framework to provide an agreed and consistent way for specifying QoS characteristics and the management of those characteristics in ODP systems.

The QoS-ODP framework defines a set of concepts and an architecture for the construction of QoS ODP systems in terms of five viewpoints. The inherent

\footnotetext{
The original version of this chapter was revised: The copyright line was incorrect. This has been
} corrected. The Erratum to this chapter is available at DOI: 10.1007/978-0-387-35585-6_68 
properties of ODP systems have several implications for the ways of handling QoS. Those are (1) the increasing need for systems that can manage QoS dynamically, (2) there is a need to understand the relationships between the end-to-end requirements for QoS characteristics and the QoS requirements that need to be imposed on individual system components so that the end-to-end requirements can be met, (3) resource management and application adaptation and policy issues.

However, several issues have to be solved in order to increase the applicability of the QoS-ODP framework. In particular, the QoS-ODP framework is not prescriptive about the use of any particular notation for the viewpoints. The viewpoint languages are abstract in the sense that they define what concepts should be supported, not how these concepts should be represented. Furthermore it has been acknowledged that a certain amount of formality in the viewpoint notations is necessary

We address in this paper the need to develop appropriate notations for the QoSaware enterprise specification of ODP systems. Currently, the UML is emerging as de-facto standard for formal object-oriented modeling. Several approaches are being used in the field of the formalization of UML. For several reasons, we use the metamodelling approach in our work. We model in UML the QoS concepts related to the enterprise viewpoint namely, the QoS foundation modelling concepts and the concepts in the QoS in enterprise specification part. The result of our analysis is a meta-model of the core QoS concepts and their relationships in the UML, which constitutes the abstract syntax of a language for QoS-aware enterprise specifications.

The remainder of the paper is organized as follows. In Section 2 we overview different approaches for formalizing the UML language. In Section 3 we describe using UML the QoS foundation modelling concepts. We describe in Section 4 the concepts of QoS in enterprise viewpoint specification. A conclusion ends the paper.

\section{MAKING UML PRECISE: METAMODELLING APPROACH}

The UML Language (Booch, 98), (Rambaugh, 99), (Warner, 98) is rapidly becoming a de-facto language for modelling object-oriented systems. Although UML provides a semantics document, an important aspect of the language is the recognition by its authors of the need to provide a precise description of its semantics. This has resulted in a Semantics Document, which is presently being managed by the Object Management Group (OMG). The approach taken is to give a meta-model description of the language. This is presented in terms of three views: the abstract syntax, wellformedness rules, and modelling elements semantics. The abstract syntax is expressed using a subset of UML static modelling notations. The well-formedness rules are expressed in OCL. Finally, the semantics of modelling elements are expressed in natural language. The task of formalizing UML has been addressed using various available formal techniques. Most of these attempts are complementary, because they approach the task from different viewpoints and aims. We will not consider the large number of papers on the semantics of UML constructs ; we overview the Precise UML (pUML) group formalisation strategy (pUML).

An approach (Gogolla, 98) shows how to use graph rewriting techniques to transform UML state machines into another simplified machine (a kind of normal form). Some papers try to formalize UML by using a particular specification language; for example, using Real-Time Action Logic, a form of real time temporal logic (Lano, 98). The relevance of the underlying model for making precise UML 
has been considered in (Breu, 98), where a different model, a kind of stream processing function is used. But the main aim is methodological: how a software engineering method can benefit from an integrative mathematical foundation.

The Precise UML (pUML) group believes that the existing UML semantics documentation and the meta-modelling approach already provide a good foundation for a precise semantics. The use of denotational semantics is the key to describing the semantics of UML precisely. UML already partially adopts the denotational approach to describe aspects of the language. The meta-modelling approach semantics (Evans, 99) naturally supports the description of denotational relationships between model elements: model elements and their denotations can both be abstracted as conceptual classes and, the relationships between them can be formalized by associations and OCL constraints. The pUML defines a formalization strategy which consists of several steps. Those steps are (1) identify specific elements that contribute to a core semantic model, (2) iteratively examine the elements seeking to verify their completeness ; here, the completeness is achieved when : (2.1) the modelling elements have a precise syntax, (2.2) are well-formed, and (2.3) have a precise denotation in terms of some fundamental aspect of the core semantic model, (3) use formal techniques to gain better insight into the existing definitions (Bruel, 98), and (4) feed the results into the UML meta-modelling, and disseminate to interested parties for feedback. Several papers have based on the pUML formalization strategy for formalizing UML constructs (Evans, 98)

As mentioned before, the RM-ODP is not perspective about the use of any particular notations and for the viewpoints. Elsewhere, any particular formal description and specification techniques is suitable for the specification of ODP systems. Recently there has been a considerable amount of research within the field of applying the UML as a formal notation to the ODP viewpoints, and particularly to the ODP enterprise viewpoint. For example, (Steen, 99), (Linington, 99) addressed how the relevant UML constructs can be used to represent the enterprise language concepts and support the enterprise specifications; a meta-model of the core concepts and their relationships is constructed. (Seteen, 99) uses UML metamodelling approach for formalizing the ODP enterprise policies; it defines an UML meta-model for policy related concepts which is built on top of the formal object oriented specification language Object-Z (step 2 of the pUML strategy).

In this respect, we use the UML metamodelling approach to define a notation for QoS-aware enterprise specifications. Our contribution is based on ideas from the field of defining notations for ODP viewpoints.

\section{QOS FOUNDATION MODELLING CONCEPTS}

The QoS-ODP framework defines QoS foundation modelling concepts, QoS in system specifications (ODP viewpoint specifications), QoS management functions, and requirements on notations in order to express QoS. QoS foundation modelling concepts are the concepts necessary in order to allow QoS characteristics and management to be expressed in ODP system specifications. QoS in ODP system specifications describe how QoS foundation modelling concepts together with the architecture concepts can be used to express QoS characteristics and management in ODP system specifications. QoS management are the functions necessary for the 
management of QoS in ODP systems and how these functions are used to meet specific QoS requirements. QoS notations describe requirements on notations for expressing ODP system specifications in order for them to be able to express QoS.

We describe in this section the QoS foundation modelling concepts and, QoS in enterprise specification. We use the UML to model the language resulting from the analysis of those concepts and their relationships.

QoS foundation modelling concepts (Figure 1) include QoS characteristics, QoS requirements, QoS relations, QoS statements and composition of QoS relations. QoS statements are QoS capabilities, QoS offers, QoS contracts and QoS requirements.

QoS characteristics are fundamental to the expression of requirements or other statements about QoS. A QoS characteristic represents some aspect of an object that can be identified and quantified. QoS characteristics are intended to be used to model the actual, rather than the observed behaviour of the objects that they characterize. QoS characteristics are delay, jitter, throughput, error, availability, reliability, integrity, confidentiality, and precedence.

QoS requirements express various things with respect to one or more QoS characteristics. Specifically, a QoS requirement is likely to express one or more values associated with the characteristics, the nature of the value, and so on.

In the context of the specification of ODP systems, a QoS requirement is applied in the sense of a requirement on the behaviour of an object. QoS requirements are expressed using the QoS relation structure. For a given object, a QoS relation states mutual obligations of an object and its environment. QoS relations (Figure 2 ) are fundamental building blocks for the expression of QoS statements (Figure 3). QoS relations are associated with individual objects.

In general, a QoS relation comprises: (1) the expectation of an object from its environment; it indicates the QoS that an object expects from its environment in order to perform its own tasks correctly and, (2) the obligation (or guarantee of QoS to the environment, i.e the QoS that each object belonging to the environment of a given object should expect from that object, this QoS will be provided by the given object as long as its environment itself performs according to object's QoS expectations. It is important to note that in some cases QoS relations can be represented by pure expectations, in which case the corresponding provision is always true; or by pure obligations, in which case the corresponding expectation is always true.

Where QoS apply to a configuration of objects, a refinement mechanism can be used in order to derive a configuration set of QoS requirements applying to the individual objects in the configuration. A set of QoS relations applying to a collection of objects is composable if and only if the conjunction of obligations logically implies the conjunction of expectations.

QoS management is the general term for any activities performed by a system or communication service to support QoS monitoring, control and administration.

QoS management activities are driven by user requirements, the systems environment, and the systems policies that are in force for the activity. User requirements are quantified and expressed as a set of QoS requirements. QoS management is carried out in accordance with the QoS policies in force for the system.

QoS capabilities are the set of QoS characteristics provided by an object. QoS capabilities can be expressed using the QoS relation structure. In the case where QoS 
capabilities apply to a configuration of objects, a refinement mechanism can be used.

QoS offers describe advertised QoS. QoS offers can apply to single objects and to configurations of objects and are expressed using the QoS relation structure. This concept is therefore applicable: 1) in the context of negotiation, 2) where QoS capabilities are partitioned into subsets which are made available as advertised QoS offers, and 3) where uncertainly exists in the knowledge of actual QoS capabilities.

A QoS contract is a contract that defines both the subsets of QoS capabilities that objects of a configuration have agreed to provide to one another and the QoS requirements they are intending to satisfy. QoS contracts can be expressed in terms of sets of QoS relations. They can either be agreed by dynamic negotiation mechanisms or be implicitly incorporated in the design of systems.

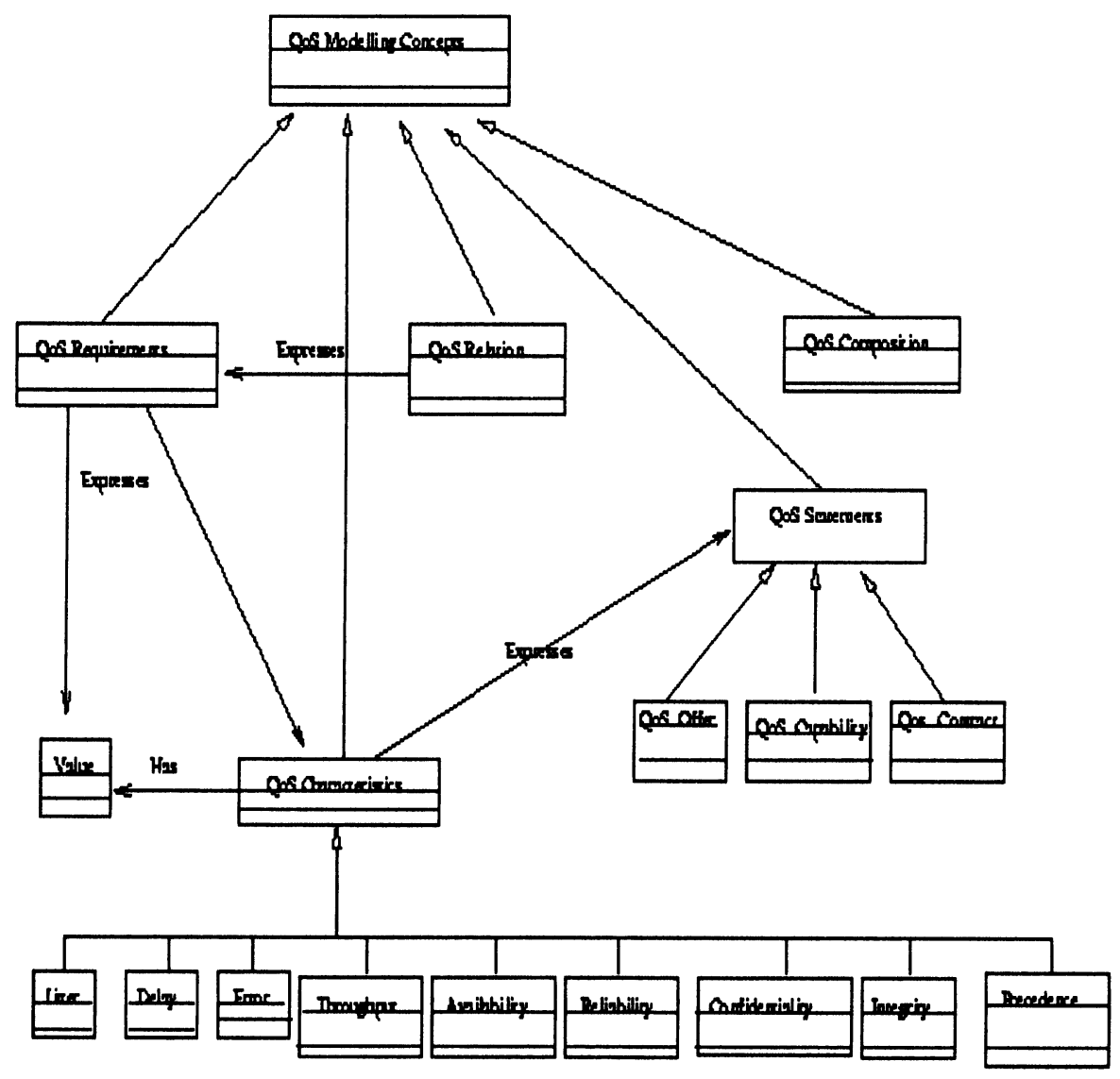

Figure 1 - Meta-Model For QoS Foundation Modelling Concepts 


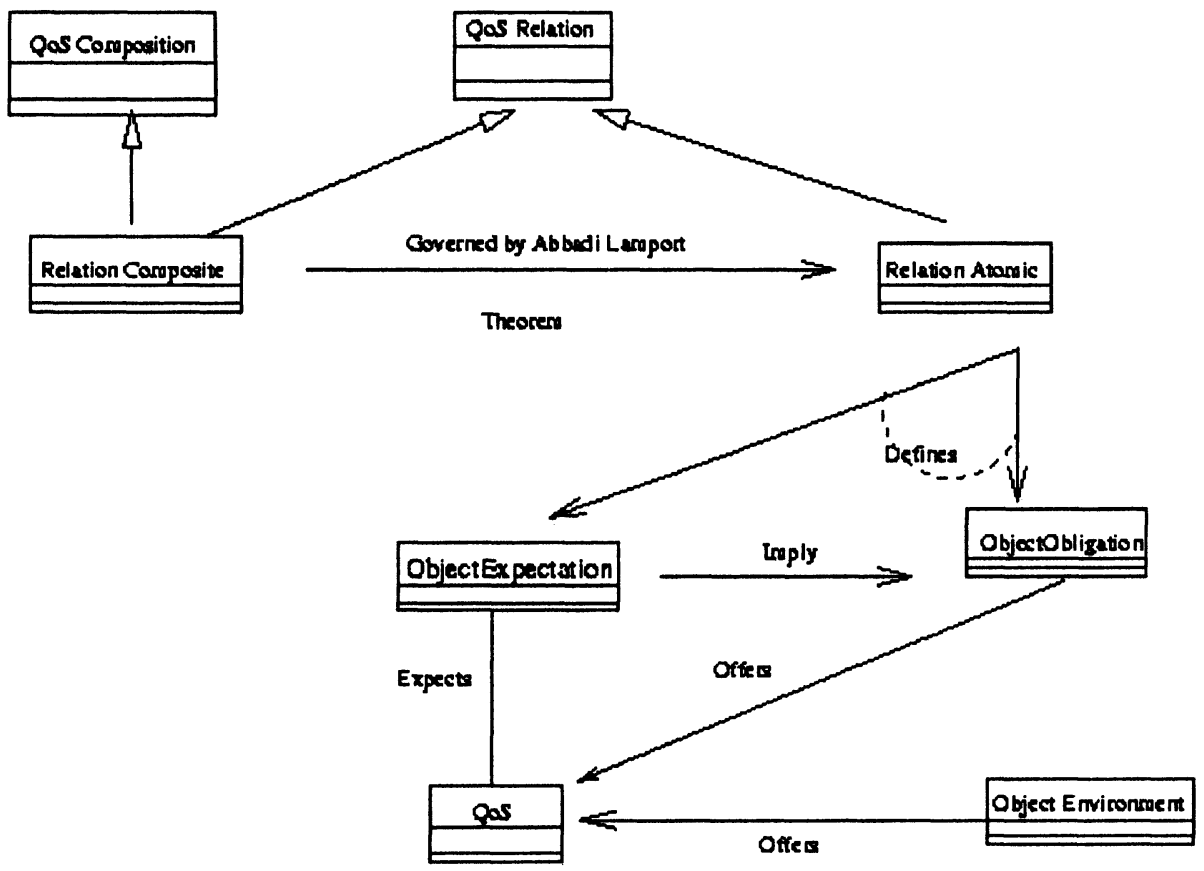

Figure 2 - A Meta-Model for QoS Relation Concept

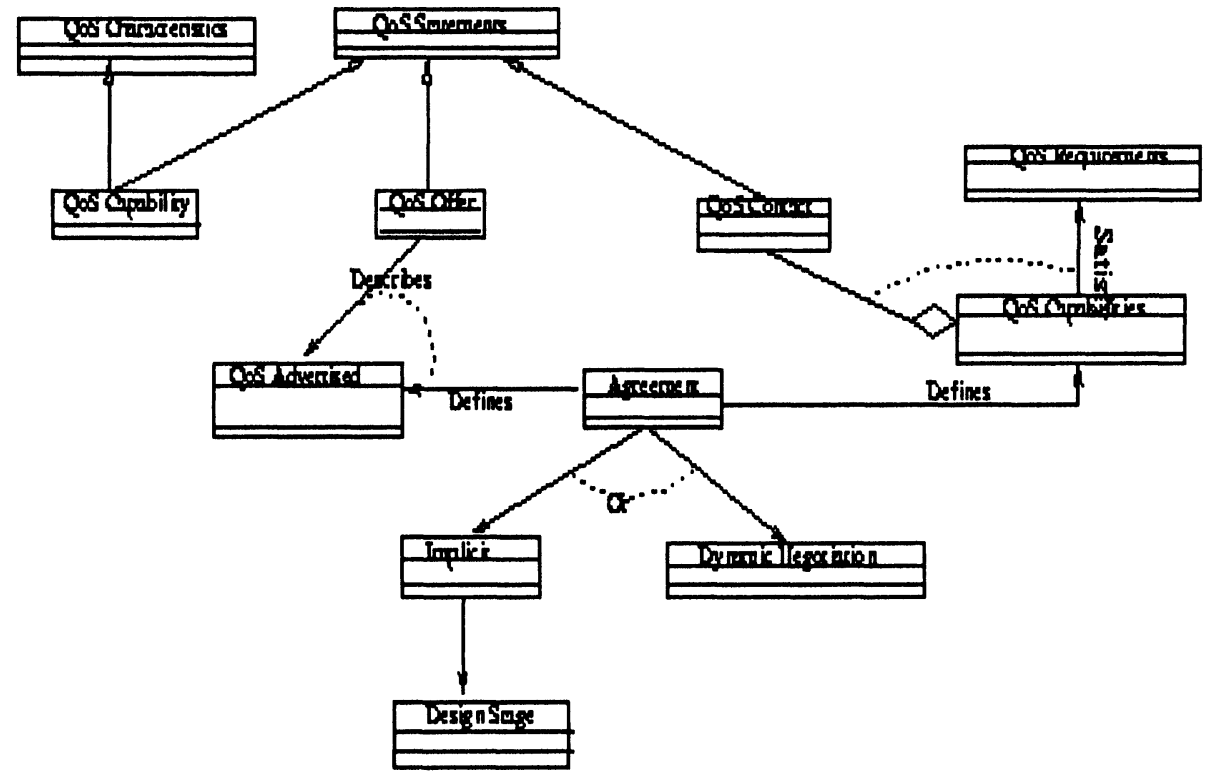

Figure 3 - A Meta-Model for QoS Statements Concept 


\section{QOS IN ENTERPRISE SPECIFICATION}

The QoS in ODP framework addresses the specification of QoS characteristics as part of the viewpoint specifications for an ODP system.

The enterprise viewpoint focuses on the QoS requirements on an ODP system. These derive from the purpose, scope and policies for that system within the environment in which it operates. The QoS requirements on an ODP system are specified by the QoS requirements on the enterprise object representing that system in a community that represents the system and its environment.

QoS requirements on an ODP enterprise object are specified in the behaviour identified by the roles that the enterprise object fulfills and the policy statements about those roles. These QoS requirements are expressed in terms of QoS relations. The behaviour specified can include negotiation of QoS contracts between client roles and server roles. This negotiation is defined in terms of statements of QoS requirements placed on a server role by a client role, statements of QoS capabilities of the client role and server role, and statements of QoS offers of the server role.

In an enterprise specification, the specification of QoS characteristics covers (1) QoS requirements on the enterprise objects and their interactions, and (2) behaviour aimed at meeting the QoS requirements and dealing with degradations in QoS.

The enterprise description also includes QoS requirements on the information defined in the information specification where those QoS requirements are related to the objectives and obligations of enterprise objects.

QoS statements in the enterprise specification are those that relate to objectives and policies of the ODP system in its environment. These statements express QoS requirements, which are taken to include requirements on the system from the outside world which can be translated into designer's requirements.

At some level of abstraction, QoS requirements are associated with community objectives and policies. These will correspond to requirements expressed at a lower level of abstraction on the enterprise objects in the community and their interactions.

\section{1 . QoS Requirements on Objects and their Interactions}

In the enterprise specification, some QoS requirements will be placed on the enterprise objects themselves, others on interactions between enterprise objects.

Those placed on enterprise objects will define requirements for QoS characteristics such as availability, reliability, safety, security, capacity etc.

Those placed on interactions or sets of interactions between enterprise objects will relate to the definition of the interactions or sets of interactions themselves. QoS requirements relating to interactions will include QoS requirements on the information obtained and QoS requirements on the interaction itself. QoS requirements on the information obtained would relate to QoS characteristics such as (1) freshness -how up-to-date the information is and, (2) precision -with what granularity the information is expressed.

QoS requirements on the interaction itself would relate to QoS characteristics such as timeliness (requirements for request/response delays), capacity (throughput), accuracy (error probability), security (access control, integrity, confidentiality, 
authentication, non-repudiation ), precedence, in the sense of the importance of the interaction relative to others -for use in cases of shortage of resources or other QoS conflicts. QoS requirements on interactions will imply QoS requirements on the enterprise objects involved in the interactions and on the environment of the objects. In some cases, for example service request/response delay, the contributions of the objects and the environment to the required QoS may be defined separately, or may be left unspecified in the enterprise specification.

Generally, QoS requirements in the enterprise specification will be expressed in terms appropriate to the level of granularity of the specification, and may serve as high-level requirement statements from which other viewpoint descriptions and conformance statements can be derived.

A QoS requirement on an enterprise object or an interaction that relates to a given characteristic must also specify what kind of QoS value is being agreed (e.g upper lower limit, target, etc.), the level of guarantee that is required, and the requirements for recovery (in terms of enterprise objects and their interactions) if the interaction or set of interactions fails, or delayed, or otherwise fails to meet the QoS requirement.

\subsection{QoS Requirements on Information}

The enterprise specification may also contain QoS requirements on the information processed by the system. These may be regarded as QoS requirements on the information model. Some relate to the information itself, others to the schemata.

The QoS requirements on the information itself include those that concern the relationships between the information in the system and the things in the real world that the information represents: for example that information in the system ODP about the position of something in space is within a certain distance of its true position. Such requirements may involve the time dimension as well. They would relate to QoS characteristics such as accuracy and completeness. QoS requirements on the static and the invariant schemata could include correctness requirements within and between information objects (the latter is sometimes termed spatial consistency). QoS requirements on dynamic schemata have similar correctness requirements. Apart from the general requirement of consistency between the different viewpoint specifications, there is a requirement that the engineering and technology specifications identify conformance points at which enterprise conformance requirements relating to $\mathrm{QOS}$ can be validated. 


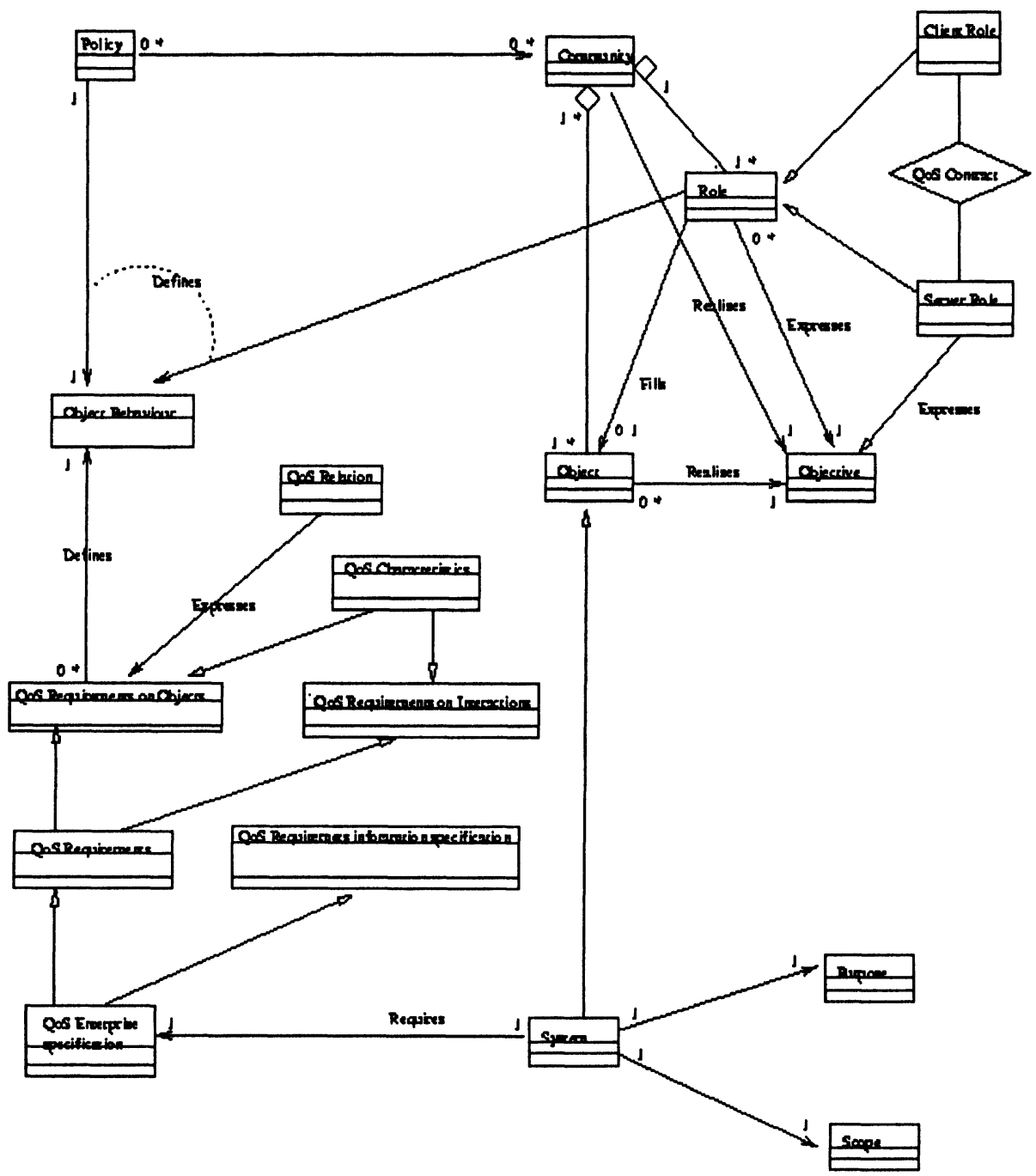

Figure 4 - Meta-Model for QoS in Enterprise Specification.

\section{CONCLUSION AND PERSPECTIVES}

The QoS-ODP standardization initiative has led to a framework by which QoS aware ODP systems can be modeled using five viewpoints. We address in this paper the need to develop formal notations for the specification of QoS-aware ODP enterprise specifications. We use the meta-modelling approach of pUML group. We define a meta-model of the core QoS concepts and their relationships in the UML, which is the syntax of a language for QoS-aware enterprise specifications. Our work constitutes the first step in the pUML strategy ; the following steps are under study.

The RM-ODP states that an environment contract may contain the specification of QoS constraints, but it does not make the nature of these constraints explicit. These constraints should reflect the capabilities present in the supporting platform. This necessitates the introduction of a resource model in the computational viewpoint. 
The resource model is a refinement of the computational viewpoint in which the computing and communication resources are made explicit. The resource model is very important for functionalities in the QoS provision phase namely, mapping, admission testing and resource reservation. We are investigating how to define the relationships between the end user requirements and the resource model. We are also investigating how to extend the QML (QoS Modelling Language) (Frolund, 98) to specify and compose the QoS relations.

\section{REFERENCES}

1. APM Ltd. ANSAware 3.0 Implementation Manual. Castle Park, Camridge CB3 ORD UK, 1991.

2. Barden R. and al. Resource ReServation Protocol (RSVP) Functional Specification. IETF RFC 1997.

3. Booch G. et al. The Unified Modelling Language Guide. Addison Wesly, 1998.

4. Breu R. et al. Systems Views and Models of UML. Physica Verlag, 1998.

5. Bruel J-M. et al. Transforming UML Models to Formal Specifications.UML'98. 1998.

6. Bulterman D. et al .Multimedia Synchronisation and Unix. Proc. 2end International Workshop on Network and Operating System Support for Digital Audio and Video, 1991.

7. Campbell A. et al. A Continuous Media Transport and Orchestration Service. SIGCOMM'92, 1992.

9. Campbell A. et al. Integrated Quality of Service for Multimedia Communications. IEEE INFOCOM'93, April 1993.

10. Coulson G. et al. Extension to ANSA for Multimedia Computing. Computer Networks.1992.

11. Coulson G. et al. Design of a QoS Controlled ATM Based Communication System in Chorus. IEEE Journal of Slected Areas in Communications, May 1995.

12. Doeringer W. et al. A Survey of Light-weight Transport Protocols for High Speed Networks.IEEE Transactions on Communications, November 1990.

13. Enterprise Viewpoint. Technical Report 8-99, Computing Laboratory, University of Kent 1999.

14. Evans A. et al. Core Meta-Modelling Semantics of UML: The pUML Approach. UML'99. 1999.

15. Evans A. et al. Developping the UML as a formal modelling notation. UML'98, 1998.

16. Feldmeir D. et al. A Framework of Architecture Concepts for High Speed Communication Systems. Computer Communication Research Group, Bellcore, 1993.

17. Frolund S. et al. QML: A language for QoS Specification. Technical Report, HP Laboratories 1998.

18. Gogolla M. et al. State Diagrams in UML- A Formal Semantics Using Graph Transformation. ICSE'98, 1998.

19. DiffServ. Differentiated Services Architecture. IETF Drafts, http://www.ietf.org/html.charters/diffserv-charter.html

20. IntServ. Integrated Services Architecture. IETF Drafts. http://www.ietf.org/html.charters/intservcharter.html.

21. ISO/IEC. Open Distributed Processing-Reference Model-Quality of Service. Technical report, ISO/IEC JTC1/SC33, July, 1998.

22. ISO/IEC. QoS Guide to Methods and Mechanisms. Technical Report, TR 13243/ X.642,1997.

23. ISO/ITU-T : RM-ODP-Part2/ Descriptive Model. Technical report, ISO/IEC DIS 10746-2 1994.

24. ISO/ITU-T. RM-ODP-Part3/ Prescriptive Model. Technical report, ISO/IEC DIS 10746-3 1994.

25. ISO/ITU-T. Basic Reference Model of Open Distributed Processing-Partl: Overview and Guide to use. Technical report, ISO/IEC CD 10746-1, July 1994.

26. Lano K. et al. Formalising the UML on Structured Temporal Theories. ECOOP'98, 1998.

27. Linington. P.F. Options for expressing ODP Enterprise Communities and their Policies by using UML. In IEEE Com. Soc. Press. EDOC'99, 1999.

28. Mullender S. Distributed Systems. 2end ed. Addison Wesley. 1993.

29. Nicolaou C. et al. An Architecture for Real Time Multimedia Systems. IEEE Journal on Selected Areas in Communications. V.8 N.3, April 1990.

30. pUML group. The Precise UML web site www.cs.york.ac.uk/puml, 1999.

31. Rumbaugh J. et al. The Unified Modelling Language Reference Manual. Addison Wesly, 1999.

32. Shulzrinne H. and al. RTP: A transport Protocol for Real-Time Applications IETF, RFC 1889, 1997.

33. Stankovic et al. Implications of Classical Schedulling Results for Real Time Systems. Addison Wesley, 1995.

34. Steen M. W. A. et al. Formalising ODP Enterprise Policies. 3rdInternational Enterprise Distributed Object Computing Conference (EDOC'99), IEEE Publishing, September 1999.

35. Warner J. et al. Object Constraint Language Addison Wesley, October, 1998. 